\title{
Point Investigation Method for Cancer Changed Tissues
}

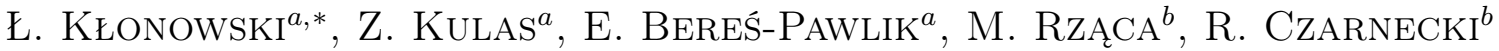 \\ AND Z. GRZEBIENIAK ${ }^{c}$
}

${ }^{a}$ Institute of Telecommunications, Teleinformatics and Acoustics, Wrocław University of Technology

Wybrzeże Wyspiańskiego 27, 50-370 Wrocław, Poland

${ }^{b}$ Oncology Department, Regional Specialistic Hospital, Kamińskiego 73a, 51-124 Wrocław, Poland

${ }^{c}$ Oncology Department, Academy Clinical Hospital, Borowska 213, 50-556 Wrocław, Poland

In this paper we describe the method of point investigation for cancer changed tissues with application of fluorescence phenomenon. The measurements have been made using a specially constructed scanning setup and fiber sensors. The experiment with investigation of endogenous fluorescence has been made on different types of slide tissues (e.g. breast and intestine tumor or precancerous and pathological skin tissues). The obtained spectral characteristics of fluorescence, with typical intensity peaks in 480-520 $\mathrm{nm}$ range, have explicitly outlined healthy and pathologically changed areas. The intensity of detected fluorescence determines the evaluation of disease advancement. Moreover, the ability to scan the surface of a tissue sample with constantly moving step of scanning setup in $X-Y$ axis allows us to present the results in a spatial distribution of fluorescence intensity.

PACS numbers: 87.57.-s, 87.57.R-, 87.57.nm, 87.19.xu, 87.19.xj

\section{Introduction}

The fluorescence is a known and used phenomenon in different fields of research. In this paper we present the exploit of one-photon excitation fluorescence. This method was used for investigation of cancer changed tissues. Cell division is a process that is present in tissues to keep the balance between cells deaths and proliferation. Some errors - mutations may occur in this process. Mutation of the genetic material of normal cells leads to carcinogenesis. Due to uncontrolled and rapid cell divisions it leads to an outgrowth of cancer above the mother cells forming a tumor (Fig. 1) [1-3].

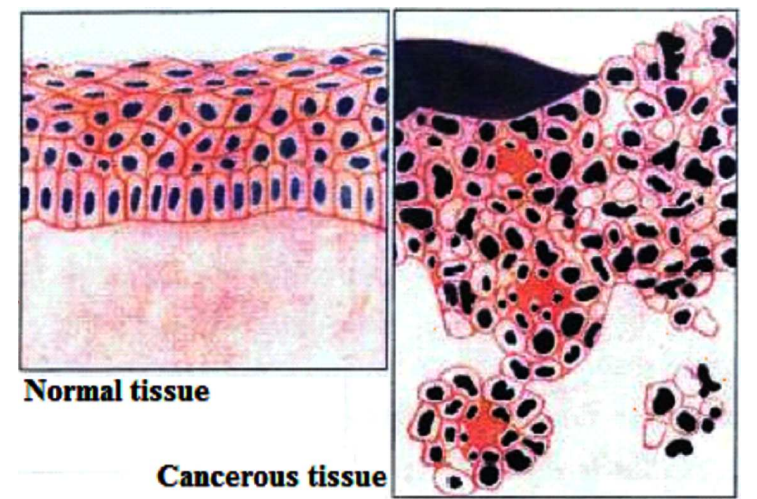

Fig. 1. Differences in structure of normal and cancerous tissue.

Changes in tissue structure causes reduction of endogenous fluophores. Low number of fluophores in cancerous

\footnotetext{
* corresponding author; e-mail: lukasz.klonowski@pwr.wroc.pl
}

tissue provides lower fluorescence intensity than in normal, healthy one. Cancer changed tissues are also supplied with more blood, and, because of high absorption of hemoglobin in about $0.3 \div 1 \mu \mathrm{m}$ range (Fig. 2), they also reduce the obtained intensity of fluorescence [4].

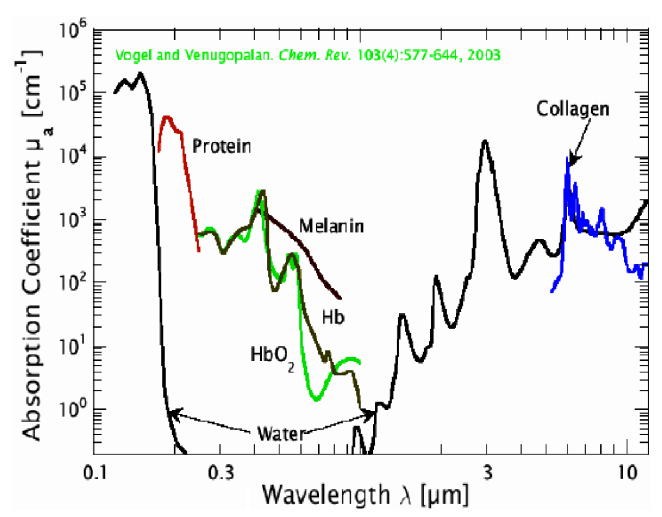

Fig. 2. Absorption coefficient for selected tissue chemical compounds [4].

In the presented method detection is possible due to differences in fluorescence intensity and it is clearly lower in pathological areas [4-8]. To obtain the best resolution and precision of measurements, the authors used a specially constructed scanning setup. This device enables positioning measurement sensors with high precision above the investigated tissue. The movement in two directions is possible thank to step motors and a micrometric $X Y$ stage. The whole setup is compatible with the Ocean Optics spectrophotometer and the software used in the experiment [1]. 


\section{Experimental setup}

The source of illumination in the experiment setup (Fig. 3) was a $404 \mathrm{~nm} \mathrm{GaN} \mathrm{laser} \mathrm{with} \approx 7.4 \mathrm{~mW}$ output power obtained at the end of a fiber sensor for fluorescence excitation. To cut any possible reflections a band-pass filter $(510 \pm 40 \mathrm{~nm})$ has been placed before the spectrophotometer.

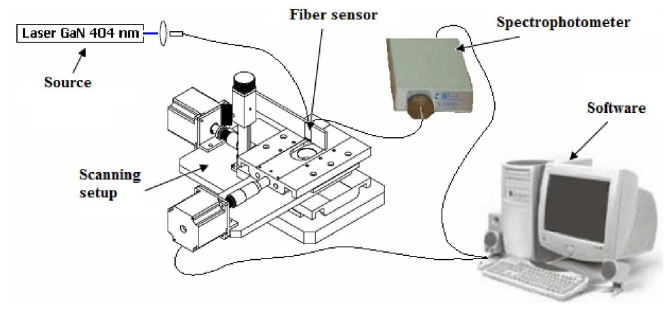

Fig. 3. Experiment setup [1].

\section{Results and discussions}

The experiment with investigation of endogenous fluorescence has been made on different types of slide tissues. Here the authors presented some of the obtained results. The first patient was a 76-year-old woman with a suspicion of skin melanoma. The size of the tissue slide cut with proper margin was about $5 \times 3 \mathrm{~cm}$ (Fig. 4).
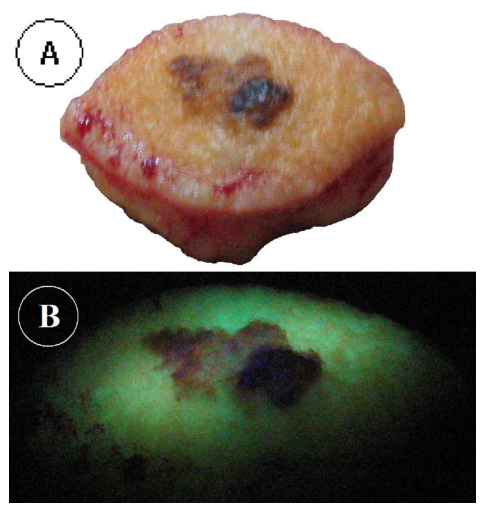

Fig. 4. Investigated tissue slide (A) and its fluorescence image $(\mathrm{B})$.

A fluorescence image of investigated tissue has been presented in Fig. 4B. It is easy to see that there is no or very low fluorescence from pathological change in the middle. The green illumination is the fluorescence of the healthy part of the tissue which corresponds to the margin. The tissue has been investigated by a point method and some of the measurement points are shown in Fig. 5A. The fluorescence intensity obtained at these points has been plotted in Fig. 5B. The closer to the center, the higher change of intensity can be observed. In the area of the biggest pathological changes the fluorescence is the lowest (points 5, 6, 7) and its value is within noise levels of the spectrophotometer used. The surrounding area (points 2, 3, 4, 8) also shows the intensity lower than that of the healthy parts of the margin which indicates an advance of disease.

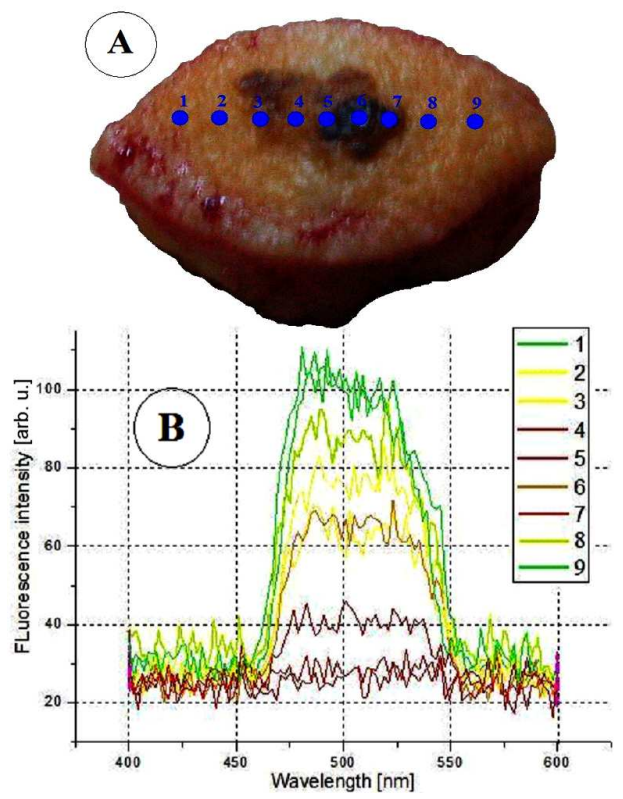

Fig. 5. Measurement points (A) and fluorescence intensity plot (B).

Additionally, the ability to scan the surface of a tissue sample with the step of scanning setup constantly moving in $X-Y$ axis allows us presenting the results in a spatial distribution of fluorescence intensity (Fig. 6). In this case the authors presented only the results of a partial scanning. The scanning has been made with a $500 \mu \mathrm{m}$ scan step, from left to right, where only areas showing pathological changes have been scanned.

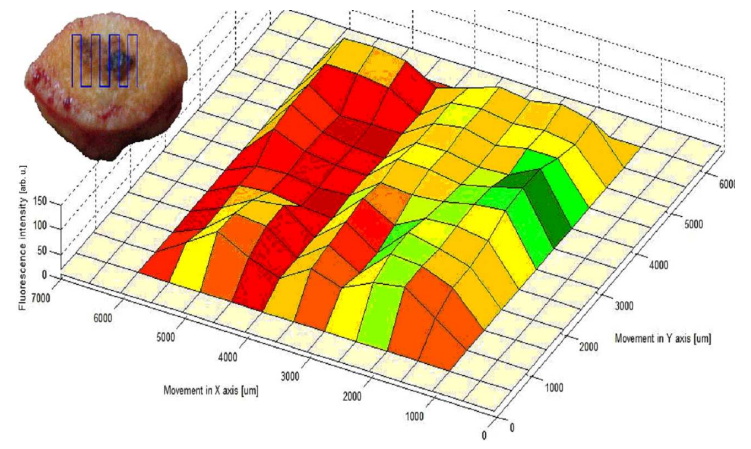

Fig. 6. Spatial distribution of fluorescence intensity.

A similar investigation has been made on an intestine slide. The patient was a 63 -year-old woman with diagnosed intestine cancer. This case required larger margin, so investigated slide was about $15 \times 13 \mathrm{~cm}^{2}$. The cancerous change is visible in the middle of the tissue (Fig. 7A). In this case the tissue has also been investigated by a 
point method, and some chosen points have been presented in Fig. 7A. The results of obtained fluorescence intensity have been plotted in Fig. 7B.

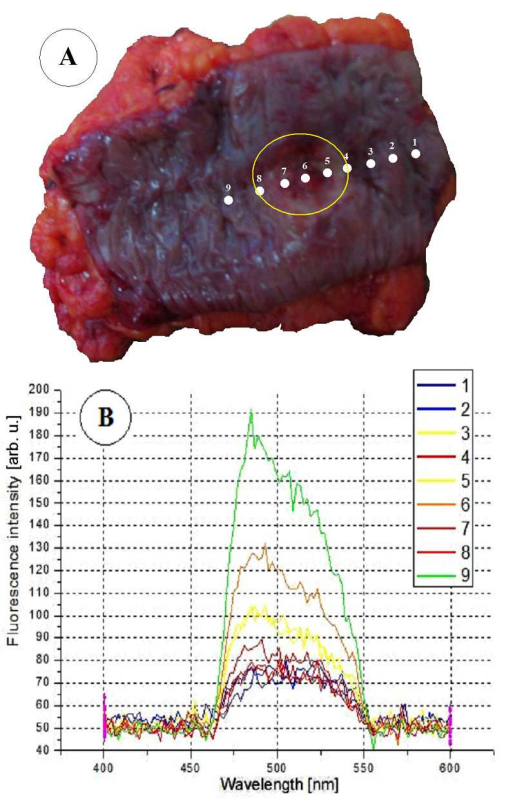

Fig. 7. Cancerous change with measurement points (A) and fluorescence intensity plot (B).

In this case low intensity characteristics for pathological changes shown are not limited to the centre of cancerous area only. Points 1, 2, 3 marked in Fig. 7A show very low intensity also in the margin. These results indicate that the cancer infiltrates the tissue beneath the surface. This situation can be overlooked during diagnose and surgery $[9,10]$. On the other side of the pathological change (point 9) the margin area shows a very high intensity of fluorescence that is characteristic for healthy tissue $[11,12]$.

\section{Summary}

All the results have been confirmed with a histopathological examination. In both cases the cancerous areas were defined correctly. Moreover, the examination confirmed that the pathological change in the intestine sample was larger, and that the infiltration of the cancer could have been missed during the diagnose and surgery. In this case the margin should be broader. The presented method can be a useful diagnostic tool for identification of cancerous areas.

\section{References}

[1] Ł. Kłonowski, E. Bereś-Pawlik, M. Rząca, R. Czarnecki, Photon. Lett. Poland 2, 52 (2010).

[2] J.A. Kiernan, M. Wessendorf, Autofluorescence: Causes and Cures, Toronto Western Research Institute, http://www.uhnres.utoronto.ca/ facilities/wcif/PDF/Autofluorescence.pdf .

[3] B. Valeur, Molecular Fluorescence - Principles and Applications, Wiley-VCH, Weinheim 2001.

[4] A. Vogel, V. Venugopalan, Chem Rev. 103, 577 (2003).

[5] H. Zeng, A. McWillimas, S. Lam, Photodiagn. Photodyn. Therapy 1, 111 (2004).

[6] E. Beres-Pawlik, K. Gasiorek, Z. Kulas, M. Rzaca, R. Czarnecki, Acta Phys. Pol. A 116, 254 (2009).

[7] S. Muc, T. Gudra, E. Beres-Pawlik, Acta Phys. Pol. A 116, 359 (2009).

[8] W. Denk, J. Strickler, W.W. Webb, Science 248, 71 (1990).

[9] G. Konieczny, Z. Opilski, T. Pustelny, E. Maciak, Acta Phys. Pol. A 116, 344 (2009).

[10] B.A. Flusberg, E.D. Cocker, W. Piyawattanametha, J.C. Jung, E.L.M. Cheung, M.J. Schnitzer, Nature Methods 2, 1173 (2005).

[11] M. Gawlikowski, T. Pustelny, B. Przywara-Chowaniec, P. Struk, Acta Phys. Pol. A 114, A-79 (2008).

[12] J.W. Lichtman, J.A. Conchello, Nature Methods 2, 1197 (2005). 